\title{
Antiphospholipid Antibody Syndrome Presenting as Subacute Abdominal Pain Due to Portomesenteric Thrombosis
}

\begin{abstract}
Thoguluva Seshadri Chandrasekar ${ }^{1}$ Bollu Janakan Gokul ${ }^{1}$ Thoguluva Chandrasekar Viveksandeep ${ }^{2}$ Kalamegam Raja Yogesh ${ }^{1}$ Suriyanarayanan Sathiamoorthy ${ }^{1}$ Menta Sanjeevaraya Prasad ${ }^{1}$
\end{abstract}

\footnotetext{
${ }^{1}$ Department of Medical Gastroenterology, MedIndia Hospitals, Chennai, Tamil Nadu, India

2SUNY Upstate Medical University, Syracuse, NY, United States
}

\begin{abstract}
Address for correspondence Thoguluva Seshadri Chandrasekar, MD, DM, FRCP, D.Sc, FACG, FASGE, MWGO, Chief Gastroenterologist, MedIndia Hospitals, No 83, Valluvar Kottam High Road, Nungambakkam, Chennai 600034, Tamil Nadu, India (e-mail: tscmedindia@yahoo.com).
\end{abstract}

J Digest Endosc

Retraction Notice

Concerning the article "Antiphospholipid Antibody Syndrome Presenting as Subacute Abdominal Pain Due to Portomesenteric Thrombosis" by Thoguluva Seshadri Chandrasekar, Bollu Janakan Gokul, Thoguluva Chandrasekar Viveksandeep, Kalamegam Raja Yogesh, Suriyanarayanan Sathiamoorthy, Menta Sanjeevaraya Prasad there is a quality concern. For this reason The Journal of Digestive Endoscopy gives notice that the paper is retracted by the Editor-in-Chief. DOI of the article is DOI: 10.1055/s-0040-1701397. 